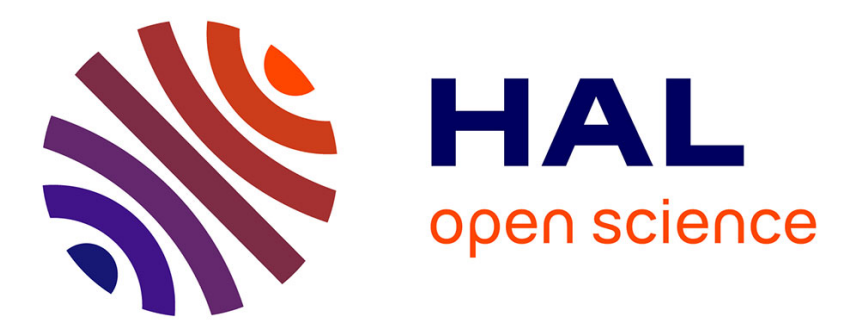

\title{
Focalisation attentionnelle appliquée aux flux auditifs. Premières mesures
}

\author{
M.-C. Botte, C. Drake, R. Brochard, S. Mcadams, A.-M. Argenti
}

\section{To cite this version:}

M.-C. Botte, C. Drake, R. Brochard, S. Mcadams, A.-M. Argenti. Focalisation attentionnelle appliquée aux flux auditifs. Premières mesures. Journal de Physique IV Proceedings, 1994, 04 (C5), pp.C5-407C5-410. 10.1051/jp4:1994585 . jpa-00253080

\section{HAL Id: jpa-00253080 https://hal.science/jpa-00253080}

Submitted on 1 Jan 1994

HAL is a multi-disciplinary open access archive for the deposit and dissemination of scientific research documents, whether they are published or not. The documents may come from teaching and research institutions in France or abroad, or from public or private research centers.
L'archive ouverte pluridisciplinaire HAL, est destinée au dépôt et à la diffusion de documents scientifiques de niveau recherche, publiés ou non, émanant des établissements d'enseignement et de recherche français ou étrangers, des laboratoires publics ou privés. 


\title{
Focalisation attentionnelle appliquée aux flux auditifs. Premières mesures
}

\author{
M.-C. BOTTE, C. DRAKE, R. BROCHARD, S. McADAMS et A.-M. ARGENTI \\ Laboratoire de Psychologie Expérimentale, Université René Descartes, URA 316 du CNRS, 28 rue \\ Serpente, 75006 Paris, France
}

\begin{abstract}
The focalization of attention on an auditory stream was studied by asking subjects to detect a temporal irregularity in the onset interval between two tones of a regular sequence in a $2 \mathrm{AFC}$ procedure. Eight subjects heard the sequence with the irregularity in the presence of two other regular sequences, each of these three sequences having its specific tempo, number and frequency of tones. One of these sequences was presented alone as a cue for the target preceding two observation intervals, each containing the three concurrent sequences. The size of the irregularity detected at a $90 \%$ level in a target sequence was only detected at chance level in both non-target sequences. Increasing the level of a probe sequence above the level of both the target and the other non-target sequence resulted in a significant improvement of the detection for five subjects. The detection in the probe was equivalent to that in the target for a 18- $\mathrm{dB}$ increase of the probe level. For the three other subjects, the detection in the probe remained at chance level whatever the probe level. Therefore, the attenuation of unattended auditory streams can attain as much as $18 \mathrm{~dB}$, at least for some subjects.
\end{abstract}

\section{INTRODUCTION}

Jusqu'à présent, un certain nombre de recherches ont comparé la détection de sons purs partiellement masqués par un bruit selon qu'il s'agissait de signaux ayant une fréquence attendue ou non. On a ainsi montré qu'un "filtre attentionnel" donné a une forme et une bande passante proches de celles du filtre auditif centré sur la fréquence attendue ; les signaux inattendus extérieurs à cette bande passante sont atténués de $7 \mathrm{~dB}[1]$. Cependant, il paraît logique de considérer que, dans les conditions naturelles d'écoute, la focalisation de l'attention s'exerce le plus souvent sur un flux auditif, c'est-àdire sur une séquence d'événements formant une suite spécifique cohérente [2]. Les expériences présentes avaient donc pour but de montrer que lorsque l'attention est focalisée sur un flux auditif, la saillance perceptive des autres flux, présents dans le même laps de temps, est amoindrie. De plus, on souhaitait évaluer l'atténuation des flux non-cibles. Nous avons modifié le paradigme sonde/cible (le "probe/signal paradigm" [3]), qui permet de mesurer la détection des sigaux attendus (cibles) et celle des signaux inattendus (sondes) pour l'adapter aux buts de cette étude.

Afin de créer trois flux auditifs, nous avons généré trois séquences sonores différentes de cadence régulière, les sons d'une séquence donnée ayant une fréquence et un tempo spécifiques et constants (Fig. 1). La durée des sons était $50 \mathrm{~ms}$ pentes comprises ; ils étaient présentés par écouteur de façon diotique à $70 \mathrm{~dB}$ SPL. La focalisation de l'attention sur un seul flux était obtenue en demandant au sujet de détecter un faible décalage temporel de l'un des sons de la séquence cible, séquence qui était par ailleurs régulière.

Trois associations "tempo/fréquence" différentes ont été retenues à la suite d'expériences préliminaires. Le tempo étant ici exprimé par la durée de l'intervalle séparant les débuts de deux sons successifs, ces associations étaient $500 \mathrm{~ms} / 784 \mathrm{~Hz}$ (séquence 1), $400 \mathrm{~ms} / 539 \mathrm{~Hz}$ (séquence 2) et 300 $\mathrm{ms} / 370 \mathrm{~Hz}$ (séquence 3 ). Les trois séquences commençaient simultanément et comportaient respectivement sept, huit et onze sons. L'ensemble avait une durée totale de $3050 \mathrm{~ms}$.

Huit sujets ont participé à trois expériences successives. 


\section{EXPERIENCE 1}

La première expérience avait pour but 1) de mesurer le seuil de détection du décalage pour chacune des séquences placées en situation de cible et 2) d'établir la valeur du décalage donnant lieu, toujours en situation de cible, à $90 \%$ de détections correctes.

\subsection{Méthodes}

Les stimuli de chaque essai (Fig. 1) comprenaient la séquence cible seule et régulière (I0) suivie de deux intervalles d'écoute (I1 et I2) dans lesquels les trois séquences étaient présentées ensemble. Le décalage, toujours situé dans la séquence cible, apparaissait alors aléatoirement en I1 ou I2. Le sujet disposait de deux boutons pour désigner l'intervalle contenant le décalage et l'allumage d'une diode verte ou d'une diode rouge l'informait en retour de l'exactitude de sa réponse. Une méthode adaptative accompagnait ce paradigme de choix forcé. Dans cette méthode, la règle de variation de la taille du décalage présenté (quatre réponses justes pour diminuer et une réponse fausse pour augmenter) permettait de déterminer le décalage correctement détecté $84,1 \%$ des fois. Chaque mesure a été répétée quatre fois pour chaque sujet pour chacune des trois séquences utilisées comme cibles, dans un ordre contrebalancé. Les deux meilleurs seuils ont été moyennés.

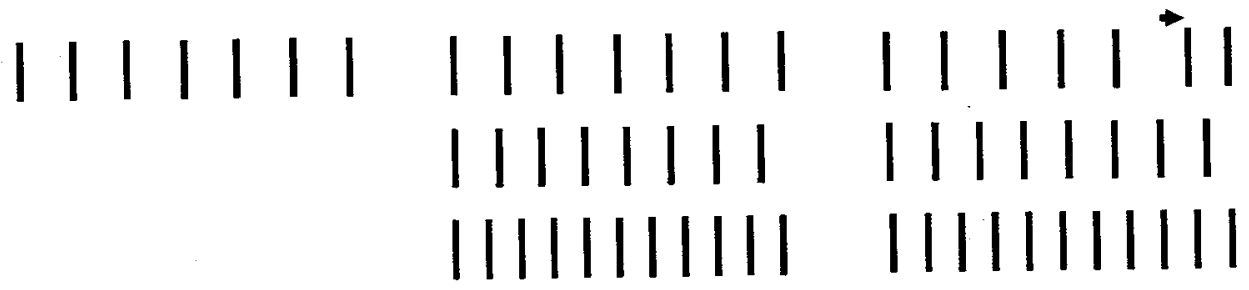

10

11

12

Figure 1. Stimuli présentés à chaque essai (le décalage à détecter est situé dans la cible).

Pour chaque sujet et chaque cible, un décalage donné, supérieur au seuil trouvé précédemment, était ensuite présenté dans des blocs de 20 essais jusqu'à l'obtention d'un taux de détections correctes situé entre 85 et $95 \%$ au cours de deux blocs successifs.

\subsection{Résultats}

La Figure 2 présente les décalages relatifs moyens obtenus ; il s'agit des décalages détectés rapportés aux tempi de référence. Pour obtenir un taux de détections correctes de $84.1 \%$ et de $90 \%$, ces décalages sont respectivement de $10,5 \%$ et $12 \%$. Les décalages détectés $90 \%$ des fois ne

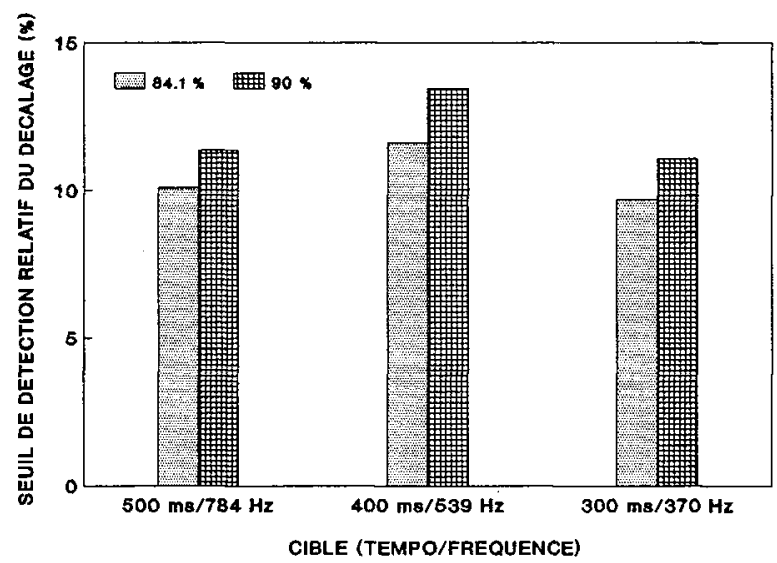

Figure 2. Décalage relatif donnant lieu à $84,1 \%$ et $90 \%$ de détections correctes 
sont pas significativement différents pour les trois cibles $(F(2,14)=3.38 ; p=0,06)$. Ces valeurs pouvaient donc servir à comparer la détection du décalage selon qu'il serait présenté dans la séquence cible ou dans une séquence non-cible.

\section{EXPERIENCE 2}

La deuxième expérience avait pour but de mesurer le pourcentage de détections correctes d'un décalage placé tantôt dans la séquence cible et tantôt dans l'une des séquences non-cibles (séquence sonde), au cours d'un même bloc d'essais.

\subsection{Méthode}

Dans cette expérience, la détection du décalage était mesurée au cours de dix blocs successifs de 20 essais. Dans un bloc donné, le décalage à détecter était maintenant situé de façon imprévisible 14 fois dans la séqence cible et 6 fois dans l'une des séquences non-cibles. Les décalages utilisés, dans la cible et les non-cibles étaient, pour chaque sujet et pour chaque séquence, ceux pour lesquels $90 \%$ de détections correctes étaient obtenus dans l'expérience précédente. D'un bloc à l'autre, la séquence cible était toujours la séquence $1(500 \mathrm{~ms} / 784 \mathrm{~Hz})$ et la séquence non-cible pouvant comporter le décalage était soit la séquence $2(400 \mathrm{~ms} / 539 \mathrm{~Hz})$, soit la séquence $3(300 \mathrm{~ms} / 370 \mathrm{~Hz})$. Le décompte du taux de réponses correctes a été établi séparément pour la cible (où il a porté sur un total de 140 présentations) et pour chacune des deux séquences non-cibles (où il a porté sur un total de 30 présentations).

\subsection{Résultats}

La Figure 3 présente les taux de détections moyens du décalage dans la cible et dans les deux autres séquences. Le pourcentage de réponses correctes s'élève à $90,3 \%$ lorsque le décalage est situé dans la cible et tombe à $59,2 \%$ et $52,5 \%$ lorsqu'il est situé dans les séquences non-cibles 2 et 3 , respectivement. Le taux de détection dans la cible (séquence 1) est significativement différent de celui observé dans l'une ou l'autre des non-cibles. On retrouve le niveau de détection attendu dans la cible (90\%) tandis que la détection ne diffère pas significativement du hasard (50\%) dans les non-cibles.

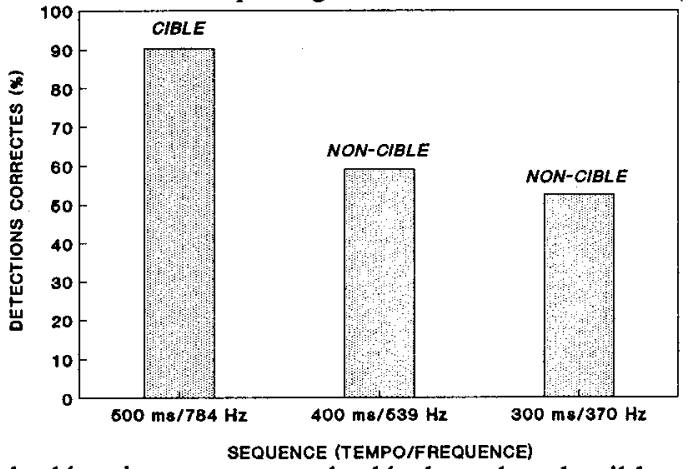

Figure 3. Pourcentage de détections correctes du décalage dans la cible et dans les non-cibles.

\section{EXPERIENCE 3}

La troisième expérience reprenait les mesures de l'expérience précédente mais en introduisant différentes augmentations de niveau des sons de la séquence sonde par rapport à celui des sons des deux autres séquences de façon à augmenter la saillance perceptive de la séquence sonde contenant parfois le décalage pour compenser l'atténuation due au "filtre attentionnel" centré sur la cible.

\subsection{Méthode}

La détection du décalage a été mesurée comme précédemment, la séquence cible était toujours la séquence $1(500 \mathrm{~ms} / 784 \mathrm{~Hz})$ contenant le décalage 14 fois sur 20, mais, cette fois, la séquence noncible où le décalage apparaissait dans six essais sur 20 était toujours la séquence $3(300 \mathrm{~ms} / 370 \mathrm{~Hz})$ appelée séquence sonde. De plus, cette séquence sonde présentait, selon les blocs, une augmentation de niveau de 6, 12, 15 ou $18 \mathrm{~dB}$ par rapport aux séquences 1 (cible) et 2 (non-cible). Les sujets passaient cinq blocs de 20 essais pour chacune de ces augmentations, dans un ordre contrebalancé. 


\subsection{Résultats}

La Figure 4A présente le pourcentage de détections correctes moyen du décalage situé dans la cible ou dans la sonde en fonction de la différence de niveau entre la sonde et la cible ; les valeurs de détection pour l'égalité de niveau entre la cible et la sonde $(+0 \mathrm{~dB})$ sont tirées de l'Expérience 2 . Ces résultats montrent que, dans la séquence cible, la détection du décalage se maintient à un taux élevé. Ce taux, proche de $90 \%$, tombe cependant à $80 \%$ lorsque le niveau de la séquence sonde atteint +15 ou $+18 \mathrm{~dB}$. En comparant les pourcentages de détections correctes obtemus dans la cible pour des niveaux croissants de la sonde, on observe que la détection dans la cible commence à être significativement diminuée à partir d'une augmentation de $15 \mathrm{~dB}$ du niveau de la sonde $(+12 \mathrm{~dB}$ vs $+15 \mathrm{~dB}: \mathrm{F}(1,7)=$ $13,09, p=0,009)$. La détection du décalage dans la séquence sonde differe du hasard dès que le niveau de la sonde est augmenté de $6 \mathrm{~dB}$. Elle est significativement plus élevée pour les niveaux intenses de la sonde $(+15$ et $+18 \mathrm{~dB})$ que pour les autres : $F(1,7)=5.743 ; p=0,04$.
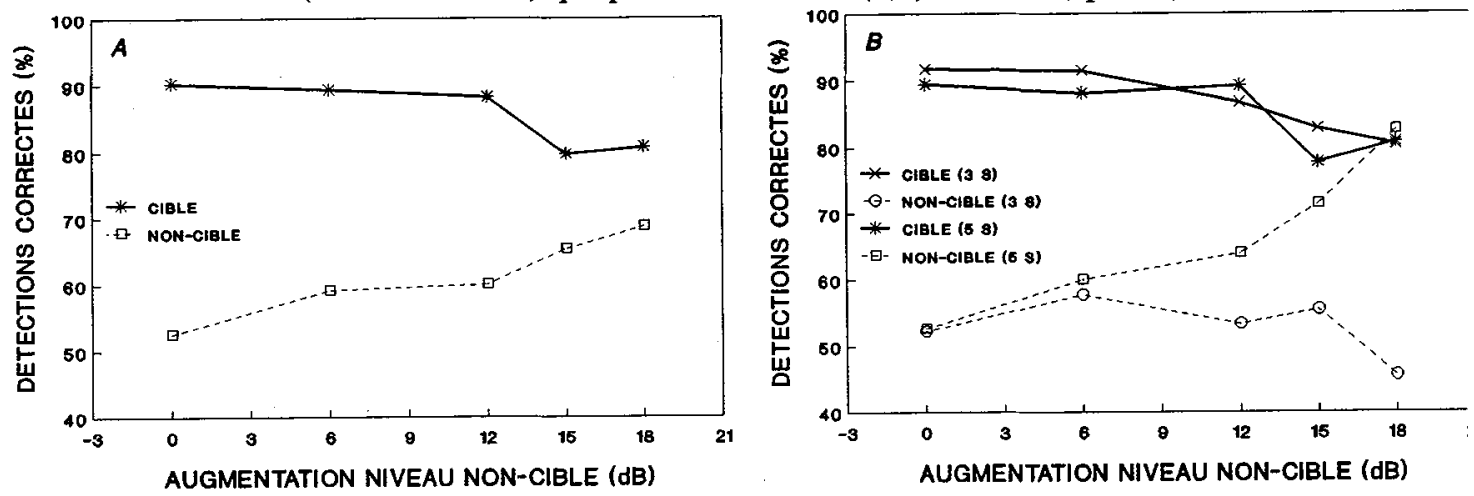

Figure 4. Pourcentage de détection correctes du décalage dans la cible et dans la sonde en fonction de l'augmentation du niveau de la sonde par rapport à celui de la cible.

Cependant les données individuelles montrent qu'il existe deux catégories de sujets basées sur la détection du décalage dans la sonde. Les données de ces deux groupes de sujets ont été moyennées séparément sur la figure 4B. Les deux groupes de sujets détectent le décalage dans la cible de façon équivalente (voir ci-dessus). En ce qui concerne la détection dans la sonde, pour cinq des sujets (5 S), la détection augmente avec l'accroissement du niveau de la sonde. Cette augmentation devient significative quand la sonde est augmentée de $18 \mathrm{~dB}$; la détection est significativement meilleure pour les deux niveaux les plus élevés par rapport aux trois autres. Pour les trois autres sujets $(3 \mathrm{~S})$, la détection dans la sonde ne varie pas significativement en fonction du niveau $(F(4,8)=2,24 ; p=$ 0,15 ) et ne differe pas du hasard.

\section{CONCLUSIONS}

L'ensemble de ces expériences montre que la focalisation de l'attention sur un flux cible s'accompagne d'une impossibilité de détecter un décalage temporel dans un flux non-écouté dont le niveau est égal à celui du flux cible (Expérience 2). De plus, pour cinq sujets sur huit, l'augmentation du niveau de la séquence non-écoutée permet d'augmenter significativement la détection dans ce flux (Expérience 3). Une augmentation de niveau de $18 \mathrm{~dB}$ de la séquence non-cible par rapport à la cible permet d'égaliser la détection dans les deux flux. On pourrait donc considérer que dans ces conditions expérimentales l'atténuation du flux non-cible due à la focalisation sur le flux cible est de $18 \mathrm{~dB}$ puisqu'elle peut être compensée par une augmentation de $18 \mathrm{~dB}$ du niveau de la séquence non-cible. Toutefois, trois sujets ne détectent pas le décalage dans le flux non-cible quelle que soit l'augmentation de niveau utilisée. L'atténuation du flux non-cible est-elle encore plus marquée chez ces auditeurs?

Les mécanismes de la détection d'un décalage temporel dans le flux non-cible de niveau augmenté demeurent cependant mal compris. De plus, les caractéristiques du stimulus utilisé nous ont montré qu'il existait de grandes différences individuelles en ce qui concerne la ségrégation perceptive de ce type de flux auditifs.

[1] Dai H., Scharf B. et Buus S., J. Acoust. Soc. Am. 89 (1991) 2837-2842.

[2] Bregman A. S., Auditory Scene Analysis (MIT Press, Cambridge, USA, 1991).

[3] Greenberg G. et Larkin W., J. Acoust. Soc. Am. 44 (1968) 1513-1523. 